\section{Child and adolescent mental health service use}

\section{HoNOSCA as an outcome measure}

\author{
M. E. GARR ALDA, P. YATES and I. HIGGINSON
}

\begin{abstract}
Background HoNOSCA (Health of the Nation Outcome Scales for Children and Adolescents) is a recently developed measure of outcome for use in child and adolescent mental health services
\end{abstract} (CAMHS).

\begin{abstract}
Aims To examine HoNOSCA's sensitivity to change, convergent validity and clinical usefulness.
\end{abstract}

Method Prospective study of new CAMHS attenders. Questionnaires completed by clinicians, parents and referrers at initial assessment and after 6 months.

Results Follow-up HoNOSCAs on 203 children indicated statistically significant change. There were significant associations between change in HoNOSCA scores, changes in other clinician- and parent-rated scales $(r=0.5 \mathrm{l}$ to 0.32 ) and in global outcome ratings by referrers, parents and clinicians. Intraclass correlation coefficients for the summated HoNOSCA scores were high. HoNOSCA change was positively correlated with initial HoNOSCA score $(r=0.46$, $P<0.00 \mathrm{I})$ and it was linked to psychiatric diagnosis.

Conclusions HONOSCA is a sensitive, valid measure of change among CAMHS attenders.

Declaration of interest The study was funded by the NorthThames Regional Health Authority.
Evidence for the effectiveness of out-patient and day patient treatments carried out in the naturalistic setting of child and adolescent mental health services (CAMHS) is limited (Jensen et al, 1996). The Health of the Nation Outcome Scales for Children and Adolescents (HoNOSCA) has been recently devised to address this problem. It is a short summary measure of the child's symptoms and social and physical functioning. Initial investigation has found it to be acceptable for use in clinical settings and to have good interrater reliability and face validity (Gowers et al, 1999). It has been shown to correlate adequately with other measures of child psychopathology, functional handicap and clinical complexity when assessing intake differences between clinics (Yates et al, 1999).

In this study we explore further the usefulness of HoNOSCA in assessing outcome in routine clinical practice in urban and suburban clinics, its convergent validity with other measures of outcome (including parent and referrer satisfaction) and its ability to measure change in relation to case complexity, frequency of clinic attendance and psychiatric diagnosis.

\section{METHOD}

\section{The clinics}

The study was carried out in two Child and Adolescent Psychiatric Out-patient Clinics in London and in a day patient clinic attached to one of these. One clinic was situated in Inner London and the other in an outlying suburban London borough. Consecutive new attenders aged 3-18 years were included. Information was obtained by questionnaires on two occasions: at initial assessment (full details of this are given in Yates et al, 1999) and at 6-month follow-up.
Clinician-completed scales used

Health of the Nation Outcome Scales for Children and Adolescents (HoNOSCA) (version 5) (children aged 3-18 years)

These rate 13 clinical features on a 5-point severity scale. Two additional questions assess parental understanding of the difficulties, and information about services. Because of the different nature of these additional two questions, as well as a total HoNOSCA score we analyse a summate clinical score and change based on the 13 clinical features only (not including parental understanding and knowledge of services) (Gowers et al, 1999).

Children's Global Assessment Scale (CGAS) (children aged 4-16 years)

This reflects the level of functioning of a child or adolescent in the previous month. It has acceptable discriminant validity and reliability (Shaffer et al, 1983; Bird et al, 1987).

Paddington Complexity Scale (PCS) (full age range)

This provides 16 items of information that would be known to professionals working within multi-disciplinary child and adolescent mental health teams. It covers three areas: psychiatric, physical/developmental and environmental. Scores in each problem area are summated into a total case complexity score and two main sub-scores (clinical and environmental). The items covered are age, gender, ethnic group, social class, diagnosis (including duration and severity), comorbidity, presence of chronic physical illness, presence of learning disability, type of schooling, who the child lives with, parental attitude to seeking help at clinics, previous contact with mental health services, and involvement with other agencies (including involvement with the Children Act) (Yates et al, 1999).

\section{Global outcome (full age range)}

At 6-month follow-up, clinicians rated on a 5-point scale the degree of improvement in the child's condition (much improved, improved, no change, worse, much worse).

\section{Parent-completed scales used Strengths and Difficulties Questionnaire (SDQ) (children aged 4-16 years)}

This is a brief behavioural screening questionnaire that provides balanced coverage 
of children's and young people's behaviour, emotions and relationships. It has adequate discriminant and predictive validity (Goodman, 1997; Goodman et al, 1998; Goodman \& Scott, 1999).

\section{Behaviour Checklist (BCL) (children aged 2-5 years)}

This 12-item checklist relates to common behaviour in young children and has wellestablished validity and test-retest reliability (Richman, 1977; Richman et al, 1982).

\section{Global outcome (full age range)}

Parents completed global measures of outcome at follow-up to indicate: changes in the child's condition (5-point scale: much improved, improved, no change, worse, much worse); changes in the parents' concern about this (4-point scale: much less concerned, less concerned, no different, more concerned); changes in their confidence in dealing with the child's problems (4-point scale: much more confident, more confident, no different, less confident); and the appropriateness of help offered at clinics (4-point scale: not at all, to some degree, very much so, completely).

\section{Scales completed by the referrer}

Global scales (full age range)

At 6-month follow-up, referrers were requested to complete questionnaires to indicate whether their referral aims had been achieved (3-point scale: yes, partly, no) and whether the intervention had been helpful to the patient and family (3-point scale: very helpful, helpful, made no difference).

\section{Training to use the scales and collection of data}

Clinic staff received training from a clinical research fellow in the use of the PCS and HoNOSCA, using case vignettes. The research fellow was available over the time of the study to help with any problems in completion or inconsistencies. At the initial assessment, parental and child questionnaires were administered just before the families attended their first clinic appointment.

For children attending at least twice, the following measures, able to reflect clinical change, were obtained at initial assessment and at 6-month follow-up: clinicians completed HoNOSCAs on all children and CGAS for children aged 4-16 years; parents completed the BCL for children aged 2-5 years and the SDQ for those aged 4-16. For all children attending we also obtained clinician-rated PCS at the initial assessment, and we sought to obtain measures on global improvement and feedback about the intervention from referrers, parents and clinicians.

Change scores on all measures were derived by subtracting for each case the 6-month follow-up data from the initial scores.

\section{RESULTS}

Descriptive information and initial case Paddington Complexity Scales were obtained for 248 subjects: the majority (229; $92 \%)$ were treated as out-patients. Fortyfive children attended only once and did not therefore qualify for repeat outcome measures. Since not all questionnaires are suitable for the same age range, the expected number of returns varies. The response rates were: clinicians completed follow-up HoNOSCAs on 203/203 cases, CGAS (4-16 years) on 191/192, global impressions of outcome on 202/203; parents completed repeat SDQs (ages 416) on 179/192, BCLs (ages 2-5) on 25/ 28, maternal General Health Questionnaires (GHQs) on 187/203; global outcome questionnaires on 189/203 attending more than once (195/248 of all attenders); referrers' global outcome questionnaires were obtained on 183/203 of those attending more than once (194/248 of all attenders).

Thirty-one clinicians from seven disciplines working within five psychiatric teams were involved in completing questionnaires as follows: psychiatry (16), nursing (4), psychology (4), social work (3), family therapy (2), speech and language therapy (1), occupational therapy (1).

\section{The sample}

Details of the sample of 248 children are given in Table 1 . The mean age of the total sample was 10 years; it contained a predominance of boys, and a comparatively large proportion (one-third) of children from ethnic minorities and from low socioeconomic groupings and broken families. About half the children had been referred by general practitioners and two-thirds of cases had diagnoses within the broad groups of disruptive/externalising or emotional/internalising disorders. Most were seen as out-patients. Sixty-one attended for fewer than three sessions, whereas 187 $(75 \%)$ attended for three or more. Table 1 shows that there were demographic differences between children attending for assessment only (fewer than three sessions) and those attending for treatment (three or more sessions). There were more children of Asian background and more children with emotional disorders in the treatment group. The mean clinic attendance was eight sessions (s.d. 8.3, range 1-54). At 6month follow-up 110 cases (44\%) were still open and $138(56 \%)$ were closed.

\section{Feasibility of using HoNOSCA in routine clinical practice to assess outcome}

We found the HoNOSCA perfectly feasible to use after training (30-60 $\mathrm{min}$ ) at initial assessment and at 6-month follow-up. It was easy and quick to use, taking between 5 and $10 \mathrm{~min}$ to complete, once clinicians were familiar with it.

\section{Reliability of HoNOSCA}

Interrater reliability was established for 15 cases which were rated by three raters presented with case vignettes. There was good interrater reliability, with intraclass correlations of over 0.82 for psychiatric symptoms, and $0.42-0.61$ for physical and social impairment, but low for knowledge about difficulties and services, $r=0.27$ and 0.03 respectively.

We also examined test-retest reliability by selecting cases for which clinicians had considered there had been no global clinical change over the 6-month period, and by comparing the initial and 6-month followup HoNOSCA scores. These were closely comparable (mean 11.58, s.d. 4.45 and mean 10.77 , s.d. 3.74$)$ with a correlation of 0.69 ( $n=53, P<0.01$, two-tailed Pearson correlation).

\section{Sensitivity of HoNOSCA and other scales in detecting clinical change}

Table 2 shows changes in HoNOSCA scores in the group attending more than once. At the 6-month follow-up there was a significant improvement, with a mean positive change of 3.61 (s.d. 4.7). There were significant changes indicating improvement on all HoNOSCA sub-scores (behaviour, impairment, symptoms, social functioning and information). Similarly significant improvements were indicated 
and to a lesser extent with parentally rated BCL $(r=0.40, P=0.04)$ and SDQ $(r=0.32$, $P<0.001)$. There was however little association with changes in maternal psychopathology as measured by the GHQ $(r=0.16, P=0.05)$.

Table 3 indicates associations between HoNOSCA change and change in the global outcome measures. There were significant associations with global impressions of positive change by clinicians, and also with global impressions of improvement by parents and with referrers' ratings on the helpfulness of the intervention for the child and family. Clinicians' and parents' global ratings of marked improvement corresponded with mean HoNOSCA changes of 6.5 and 5.6 respectively (some improvement corresponded with HoNOSCA change scores of 4). Judgements by referrers of marked helpfulness to families reflected a mean HoNOSCA change of 4.6. These were based on only 154 cases, since referrers of the other cases had not seen the family following contact with Child Psychiatric Clinics and could not therefore express an opinion on this.

\section{HoNOSCA change in relation to initial HoNOSCA severity and case complexity}

We examined whether HoNOSCA change was associated with initial case severity on the HoNOSCA. We found a significant positive correlation $(r=0.468, P<0.001$, $n=215$ ), indicating more change with more initial severity.

We also explored whether HoNOSCA change was associated with more initial case complexity. We had assessed the latter using the Paddington Complexity Scale (PCS). This provides information on a number of clinical and demographic items, as described above. Individual items are scored by the degree to which they are likely to add complexity to case management. They are summated into total case complexity scores, and into sub-scores (i.e. psychiatric and physical/developmental sub-scores, which are again summated into a clinical complexity sub-score, and an environmental complexity sub-score). We found no association between changes in HoNOSCA score and PCS total complexity score, nor between HoNOSCA change and psychiatric, developmental, clinical or environmental complexity sub-scores. We then examined whether change in HoNOSCA score was linked to any of the 
Table 2 Changes in HoNOSCA and other questionnaire scores in children attending two or more times

\begin{tabular}{|c|c|c|c|c|c|c|}
\hline & \multicolumn{2}{|c|}{ Assessment } & \multicolumn{2}{|c|}{ 6-month follow-up } & \multirow[t]{2}{*}{ Change in score } & \multirow{2}{*}{$\begin{array}{c}t \text {-test } \\
P \text { values }\end{array}$} \\
\hline & mean & (s.d.) & mean & (s.d.) & & \\
\hline \multicolumn{7}{|l|}{ HoNOSCA $(n=203)$} \\
\hline Clinical score' & 11.40 & $(4.89)$ & 7.79 & $(4.93)$ & 3.61 & $<0.001$ \\
\hline Total score & 12.95 & $(5.68)$ & 8.60 & $(5.43)$ & 4.35 & $<0.001$ \\
\hline \multicolumn{7}{|l|}{ Subgroups } \\
\hline Behaviour & 3.15 & $(2.07)$ & 1.88 & $(1.73)$ & 1.27 & $<0.001$ \\
\hline Impairment & 1.56 & $(1.56)$ & 1.18 & (1.39) & 0.38 & $<0.001$ \\
\hline Symptoms & 2.22 & $(1.83)$ & 1.45 & $(1.37)$ & 0.77 & $<0.001$ \\
\hline Social functioning & 4.43 & $(2.72)$ & 3.31 & $(2.58)$ & 1.12 & $<0.001$ \\
\hline Information & 1.55 & $(1.92)$ & 0.76 & $(1.22)$ & 0.79 & $<0.001$ \\
\hline $\operatorname{CGAS}(n=191)$ & 53.92 & $(10.90)$ & 60.93 & (12.59) & -7.03 & $<0.001$ \\
\hline BCL $(n=24)$ & 11.30 & $(4.80)$ & 9.25 & $(4.55)$ & 2.04 & 0.009 \\
\hline SDQ $(n=172)$ & 19.84 & $(6.69)$ & 16.86 & $(6.98)$ & 2.98 & $<0.001$ \\
\hline Parental GHQ $(n=149)$ & 5.68 & $(6.54)$ & 4.11 & (5.59) & 1.56 & $<0.001$ \\
\hline
\end{tabular}

I. Clinical score $=$ total score without the information subgroup.

HoNOSCA, Health of the Nation Outcome Scales for Children and Adolescents; CGAS, Children's Global Assessment

Scale; SDQ, Strengths and Difficulties Questionnaire; BCL, Behaviour Checklist; GHQ, General Health Questionnaire.

individual demographic or complexity items documented by the PCS. The only significant association was with the severity of the clinical picture (mean changes of 0.52 for mild cases, 3.7 for moderate and 4.4 for severe cases, ANOVA $P=0.009$ ). At a level short of statistical significance, learning disability was linked to less HoNOSCA improvement (mean HoNOSCA change of 4.0 in those without and 2.8 in those with learning disability, $t$-test, $P=0.07$ ).

\section{Change in HoNOSCA score, and length of treatment}

We found little association between the number of sessions attended and change in HoNOSCA $(r=0.18, P=0.01)$. There was similarly little association between the number of sessions attended and change on the CGAS $(r=0.20, P=0.004)$, SDQ $(r=0.17, \quad P=0.02), \quad$ BCL $\quad(r=-0.03$, $P=0.8)$ and maternal GHQ $(r=0.17$, $P=0.03$ ).
There was however a modest correlation between initial HoNOSCA rating and the number of sessions attended at clinics $(r=0.31, P<0.001)$. We examined this issue further by comparing the HoNOSCA change in cases attending for assessment only (defined here as attending twice, as we did not collect repeat measures on those attending once only) and in those attending for treatment (three or more sessions). Table 4 shows that there were no differences between the groups in the degree of change in HoNOSCA, CGAS and SDQ. There were no differences between the groups in global outcome measures (i.e. improvement, concern and confidence regarding the problem). However in the treatment group, significantly more parents thought they had received appropriate help and significantly more referrers felt that the aims of their referrals had been achieved and the intervention had been helpful to the child and family.

\section{HoNOSCA change and psychiatric diagnosis}

There were significant differences between diagnostic groups in HoNOSCA initial scores and changes in score (Table 5). The biggest change in HoNOSCA score occurred in the child with a psychotic disorder. Otherwise, the greatest HoNOSCA changes were seen in children with mood disorders and adjustment or stress disorders; and the smallest changes in those with psychosomatic and eating disorders.

Table 3 Mean change in HoNOSCA score and change in global outcome measures

\begin{tabular}{|c|c|c|c|c|c|}
\hline Clinicians' global assessment of change' & Much improved & Improved & No change & Worse & Much worse \\
\hline HoNOSCA change & $6.55(n=44)$ & $4.01(n=100)$ & $0.82(n=5 I)$ & $-1.00(n=7)$ & $0(n=0)$ \\
\hline Parental global assessment of change ${ }^{2}$ & Much improved & Improved & No change & Worse & Much worse \\
\hline HoNOSCA change & $5.62(n=37)$ & $4.01(n=87)$ & $2.29(n=52)$ & $0.50(n=10)$ & $0.50(n=2)$ \\
\hline Referrers' assessment of helpfulness to family ${ }^{3,5}$ & Very helpful to family & Helpful to family & Made no difference & \multicolumn{2}{|c|}{ Made the problem worse } \\
\hline HoNOSCA change & $4.67(n=36)$ & $3.80(n=95)$ & $1.90(n=20)$ & \multicolumn{2}{|l|}{$9.00(n=I)$} \\
\hline
\end{tabular}

\begin{tabular}{|c|c|c|c|}
\hline $\begin{array}{l}\text { Referrers' assessment of whether referral aims } \\
\text { were } \text { met }^{4}\end{array}$ & Referral aims achieved & Referral aims partly achieved & Referral aims not achieved \\
\hline HoNOSCA change & $5.02(n=55)$ & $3.49(n=80)$ & $1.53(n=19)$ \\
\hline
\end{tabular}

\section{ANOVAs:}

I. $P<0.001$

2. $P=0.001$.

3. $P=0.09$.

4. $P=0.01$.

5. Two questions were not completed by the referrers.

HoNOSCA, Health of the Nation Outcome Scales for Children and Adolescents. 
Table 4 Length of treatment (assessment $v$. treatment) in relation to symptom change and parents' and referrers' views about treatment

$\begin{array}{ccc}\text { Assessment } & \text { Treatment } & \text { Statistics } \\ \text { (I or } 2 \text { attendances }) & (\geqslant 3 \text { attendances }) & \end{array}$

$\begin{array}{llcl}\text { Symptoms as measured by questionnaires completed by clinicians and parents (mean and s.d.) } & t \text {-test } \\ \text { HoNOSCA change } & 3.19(5.15)(n=16) & 3.63(4.62)(n=187) & P=0.7 \\ \text { CGAS change } & 6.19(8.89)(n=16) & 7.05(11.62)(n=175) & P=0.7 \\ \text { SDQ change } & 2.50(5.17)(n=14) & 3.06(5.02)(n=158) & P=0.6\end{array}$

Parents' and referrers' views about treatment

\begin{tabular}{lccc} 
Parents' views & $(n=35)$ & $(n=174)$ & \\
$\begin{array}{l}\text { Appropriateness of treatment } \\
\text { Completely }\end{array}$ & $5(14 \%)$ & $25(14 \%)$ & \\
Very much & $12(35 \%)$ & $63(37 \%)$ & $\chi^{2}=14.2$ \\
To some degree & $11(31 \%)$ & $80(46 \%)$ & d.f. $=3$ \\
Not at all & $7(20 \%)$ & $6(3 \%)$ & $P=0.003$ \\
& & & \\
Referrers' views & $(n=56)^{1}$ & $(n=168)$ & \\
Referral aims & & & \\
Achieved & $13(24 \%)$ & $49(29 \%)$ & $\chi^{2}=8.8$ \\
Partly achieved & $15(27 \%)$ & $77(45 \%)$ & d.f.=2 \\
Not achieved & $12(21 \%)$ & $16(10 \%)$ & $P=0.01$ \\
Unknown as no contact & $16(28 \%)$ & $26(16 \%)$ & \\
& & & \\
Helpfulness to patient & $(n=57)$ & $(n=167)^{1}$ & \\
Very helpful & $4(7 \%)$ & $33(20 \%)$ & \\
Helpful & $10(17 \%)$ & $41(24 \%)$ & $\chi^{2}=15.6$ \\
Of some help & $12(21 \%)$ & $50(29 \%)$ & d.f.=4 \\
Made no difference & $15(27 \%)$ & $16(10 \%)$ & $P=0.003$ \\
Made the problem worse & $0(0 \%)$ & $1(1 \%)$ & \\
Unknown as no contact & $16(28 \%)$ & $26(16 \%)$ & \\
\hline
\end{tabular}

I. One question was not completed by the referrers.

HoNOSCA, Health of the Nation Outcome Scales for Children and Adolescents; CGAS, Children's Global Assessment Scale; SDQ, Strengths and Difficulties Questionnaire.

To determine whether the lack of associations in the total group between HoNOSCA change and PCS case complexity was masking differential associations in individual diagnostic groups, we analysed PCS total scores and sub-scores by diagnosis; and we then examined correlations between HoNOSCA change and complexity scores in each individual diagnostic group.

Table 5 shows that there were significant differences between diagnostic groups on both total complexity scores and complexity sub-scores. Physical/developmental complexity was highest for children with conduct, hyperkinetic and 'other' disorders (which included autistic-spectrum disorders). The highest environmental complexity was found in the conduct and psychosomatic disorders. However, we failed to find significant correlations between HoNOSCA change and physical/ developmental or environmental complexity for any of the diagnostic groups. The only associations found were between HoNOSCA change and psychiatric complexity (which, as well as diagnosis, includes items related to clinical severity, duration and comorbidity), indicating more change with more complexity or clinical severity in mood disorders $(r=0.39, P=0.04$, $n=26)$ and in conduct disorders $(r=0.33$, $P=0.01, n=57)$.

We also examined whether a change in HoNOSCA score might be differentially associated with length of treatment in individual diagnostic groups. The only significant correlation was found in the mood disorder group ( $r=0.46, P=0.01, n=26$ ).

\section{DISCUSSION}

In this study we have found HoNOSCA to be an easy-to-use, reliable and sensitive tool for clinicians to assess clinical change in children and adolescents attending psychiatric clinics. Its validity was indicated by the significant associations with other measures of change made by a variety of informants, including parents and those referring the children to clinics. Its reliability was good, except for the last two items on parental knowledge about the condition and services, which we therefore excluded from our analysis.

In line with previous recent reports about the use made of child and adolescent mental health clinics (Hoare et al, 1995; Gowers et al, 1999) most parents, referrers and clinicians felt that the children had improved significantly following contact with clinics. Parents also reported benefits to their parenting of the children's difficulties. Marked improvements in parents' and referrers' global outcome measures were associated with HoNOSCA change scores of 45. A slightly more demanding change of six HoNOSCA points reflected clinician-rated global judgements of marked improvement. This is similar to the findings by Gowers $e t$ al (1999), where a change of 7.7 HoNOSCA points was associated with clinicians' retrospective assessment of a child being much better. This level of change could therefore be used as a guide or anchor-point for child psychiatric clinics wishing to audit clinical change among their clientele. It is important to highlight, however, that these changes were obtained at 6-month follow-up, when only half the cases had been closed. They do not therefore necessarily indicate improvement on discharge from clinics.

The HoNOSCA changes described in this and other papers (Gowers et al, 1998, 1999) are more substantial than those derived from the use of its parent scale (HoNOS or Health of the Nation Outcome Scales) in adult psychiatric patients (Sharma et al, 1999). This indicates that the measure may be particularly helpful for use in child and adolescent psychiatric clinics.

The use of HoNOSCA allowed the study of a variety of clinical and service features. Thus we found associations between HoNOSCA-measured improvement, high initial severity of the clinical condition and individual psychiatric diagnoses. We failed, however, to find associations with initial case complexity or frequency of clinic attendance. 


\begin{tabular}{|c|c|c|c|c|c|c|c|}
\hline \multirow[t]{2}{*}{ Diagnostic group } & \multicolumn{2}{|c|}{ HoNOSCA } & \multicolumn{5}{|c|}{ Complexity' $(n=248)$} \\
\hline & Initial $(n=248)$ & Change $(n=203)$ & Psych & Phys/dev & Clinical & Environ & Total \\
\hline No diagnosis $(n=39)$ & $7.46(4.40)$ & $2.37(3.27)$ & $2.49(1.10)$ & $0.59(0.99)$ & $3.08(1.74)$ & $2.10(1.87)$ & $5.15(2.88)$ \\
\hline Stress reaction/adjustment $(n=28)$ & $9.89(4.68)$ & $5.10(3.53)$ & $3.64(0.95)$ & $0.39(0.74)$ & $4.04(1.14)$ & $2.54(2.15)$ & $6.57(2.44)$ \\
\hline Anxiety $(n=25)$ & II. $20(4.60)$ & $3.48(4.98)$ & $5.68(1.07)$ & $0.76(1.27)$ & $6.44(1.78)$ & $2.12(1.54)$ & $8.56(2.95)$ \\
\hline Depression $(n=28)$ & II.68 (5.24) & $5.27(5.94)$ & $5.29(1.05)$ & $0.50(0.92)$ & $5.79(1.50)$ & $2.18(1.61)$ & $7.96(2.56)$ \\
\hline Hyperkinesis $(n=33)$ & II.42 (4.24) & $3.65(4.20)$ & $6.97(0.85)$ & $0.94(1.06)$ & $7.91(1.16)$ & $2.27(1.77)$ & $10.18(2.47)$ \\
\hline Conduct $(n=70)$ & $12.77(5.19)$ & $3.28(4.49)$ & $7.54(1.58)$ & $\mathrm{I} .06(\mathrm{I} .4 \mathrm{I})$ & $8.59(2.38)$ & $3.37(2.23)$ & $11.96(3.83)$ \\
\hline Psychosis $(n=2)$ & $22.50(2.12)$ & 13.00 & $8.50(0.71)$ & $0.50(0.7 \mathrm{I})$ & $9.00(0.00)$ & $1.50(2.12)$ & $10.50(2.12)$ \\
\hline Statistics ANOVA & $P<0.00 I$ & $P=0.04$ & $P<0.001$ & $P=0.1$ & $P<0.001$ & $P=0.009$ & $P<0.00 I$ \\
\hline
\end{tabular}

I. Complexity as measured by the Paddington Complexity Scale which has the following subgroups: Psych, psychiatric; Phys/dev, physical/developmental; Clinical, psychiatric+ physical/developmental; Environ, environmental; Total, clinical+environmental.

HoNOSCA, Health of the Nation Outcome Scales for Children and Adolescents.

\section{Change in HoNOSCA score and clinical severity}

Change in HoNOSCA score was correlated with high initial HoNOSCA score and with high ratings of the severity of the condition on the Paddington Complexity Scale. The study was conducted in secondary psychiatric out-patient services where patients had initial mean HoNOSCA scores of 11.40 (s.d. 4.89). These services may be expected to attend to the more severe and complex mental health problems of children and adolescents, and our findings may not be applicable to other units. HoNOSCA may thus be less sensitive in detecting change in milder cases seen by singlediscipline community units or in primary care, but may be particularly helpful in in-patient settings.

\section{HoNOSCA change and case complexity}

Perhaps surprisingly, HoNOSCA change was not linked to initial case complexity on the PCS, nor was it associated with the variety of variables making up the environmental complexity score or with the demographic variables examined. It had seemed logical to assume that single parenthood, 'at-risk' status, a non-facilitative attitude by parents, associated medical and educational problems, ethnicity, age and gender would have an adverse influence on the implementation of treatments and thus on outcome. A possible explanation for our negative findings is that clinics were so adept at dealing with these extraneous factors that they were able to make sure that they did not work against the treatment: clinicians would successfully adapt the nature and intensity of their intervention to the complexity of the case. Alternatively, the treatments put into place would have been so powerful as to not be adversely influenced by these factors. It is also possible, however, that change in the HoNOSCA score was a naturally occurring phenomenon, independent of clinic use, and that the assumed complexity factors simply do not affect the collective natural outcome of these disorders.

\section{Associations with length of treatment}

The lack of associations between the number of sessions attended and HoNOSCA change could be taken to indicate that the interventions at clinics had been inconsequential for the improvement noted. However, the positive associations found between initial HoNOSCA-rated severity and the number of sessions attended, and the significant correlations between change in HoNOSCA and length of treatment in the mood disorder group suggest that clinics may have been skilled at providing the number of sessions required for individual problems. More parents and referrers felt the children had improved following attendance and that the help given was appropriate and had met the referrers' aims, when children had attended for treatment rather than for assessment only. In parents' and referrers' view there seemed to be a connection between improvement and clinic intervention.

\section{Change in relation to psychiatric diagnosis}

There were differences in the changes in HoNOSCA scores in individual diagnostic groups, though this did not simply follow diagnostic predictions. Conduct disorder, anxiety disorders and hyperkinesis improved by similar amounts, the greatest change being seen in a child with a psychotic disorder. However, in line with expectations, children with stress and mood disorders improved most, indicating that the work of the clinics with these disorders may result in the largest HoNOSCA change. Eating disorders improved little, which may have been related to the particularly low initial HoNOSCA scores. Children with psychosomatic disorders were poor responders, and interestingly had the highest environmental complexity scores. The use of HoNOSCA to audit work at 
clinics may therefore helpfully monitor individual conditions separately.

\section{Need for training}

It should be pointed out that the results of this study were based on HoNOSCA ratings made by clinicians who had been carefully trained in its use. The importance of scoring HoNOSCAs in a standardised way and of incorporating regular training into the audit cycle has rightly been emphasised by those developing the scales (Gowers et al, 1998, 1999).

\section{REFERENCES}

Bird, H. R., Canino, G., Rubio-Stipec, M., et al (1987) Further measures of the psychometric properties of the Children's Global Assessment Scale. Archives of General Psychiatry, 44, 821-824.

Goodman, R. (1997) The Strengths and Difficulties Questionnaire: a research note. Journal of Child Psychology \& Psychiatry, 38, 58I-586.

_ , Meltzer, H. \& Bailey, V. (1998) The Strengths and Difficulties Questionnaire: a pilot study on the validity of the self-report version. European Child and Adolescent Psychiatry, 7, 125-130.

— \& Scott, S. (1999) Comparing the Strengths and Difficulties Questionnaire and the Child Behaviour Checklist: is small beautiful? Journal of Abnormal Child Psychology, 27, 17-24.

Gowers, S. G., Harrington, R. C. \& Whitton, A. (1998) Health of the Nation Outcome Scales for Children and Adolescents (HoNOSCA). London: Royal College of Psychiatrists' Research Unit.

_, _ _, et al (1999) Brief scale for measuring the outcomes of emotional and behavioural disorders in children. Health of the Nation Outcome Scales for Children and Adolescents (HoNOSCA). British Journal of Psychiatry, I74, 413-416.

Hoare, P., Scarth, L. \& Forbes, F. (1995) Audit of the Edinburgh Child Psychiatry Out-Patient Service. ACPP Review \& Newsletter, 17, 139-148.

Jensen, P. S., Hoagwood, K. \& Petti, T. (1996) Outcomes of mental health care for children and adolescents: II. Literature review and application of a comprehensive model. Journal of the American Academy of Child \& Adolescent Psychiatry, 35, 1064-1077.

\section{CLINICAL IMPLICATIONS}

- HoNOSCA is a sensitive, valid measure of change in those attending child and adolescent psychiatric clinics. It can be recommended to audit service use.

- Greater HoNOSCA change correlated with higher initial HoNOSCA scores. HoNOSCA may be most useful to assess change in severely affected cases.

- HoNOSCA change varies according to individual diagnostic groupings.

\section{LIMITATIONS}

The study was carried out in clinics in urban and suburban areas, drawing patients with high levels of psychosocial disadvantage.

- This is not a study of treatment efficacy: changes cannot necessarily be ascribed to clinic intervention, and may reflect spontaneous improvement.

- The outcomes indicated changes 6 months following initial assessment, when only half the cases had been discharged. They do not therefore indicate outcome on discharge.

M. E. GARRALDA, MD, Professor of Child and Adolescent Psychiatry, P. YATES, MRCPsych, Clinical Research Fellow, Academic Unit of Child and Adolescent Psychiatry, Imperial College School of Medicine and Dentistry, London; I. HIGGINSON, PhD, Professor of Palliative Care and Policy, King's College School of Medicine and Dentistry and St Christopher's Hospice, London

Correspondence: P. Yates, Clinical Research Fellow, Academic Unit of Child and Adolescent Psychiatry, Imperial College School of Medicine, St Mary's Campus, Norfolk Place, London W2 IPG, UK

(First received 20 September 1999, final revision 18 January 2000, accepted 19 January 2000)

Richman, N. (1977) Is a behaviour checklist for preschool children useful? In Epidemiological Approaches in Child Psychiatry (ed. P. J. Graham). London: Academic Press.

—, Stevenson, J. \& Graham, P. J. (1982) Pre-School to School. A Behavioural Study. London: Academic Press.

Shaffer, D., Gould, M. S., Brasic, J., et al (1983) A Children's Global Assessment Scale (CGAS). Archives of General Psychiatry, 40, 1228-1231.
Sharma, V. K., Wilkinson, G. \& Fear, S. (1999) Health of the Nation Outcome Scales: a case study in general psychiatry. British Journal of Psychiatry, 174, 395-398.

Yates, P., Garralda, M. E. \& Higginson, I. (1999) Paddington Complexity Scale and Health of the Nation Outcome Scales for Children and Adolescents. British Journal of Psychiatry, 174, 417-423. 